\title{
Macroscopic pairing vibrational model, self-consistent pairing coupling constant, and the fifth term of von Weizsäcker's semiempirical formula
}

\author{
C. H. Dasso and M. I. Gallardo \\ Departamento de Física Atómica, Molecular y Nuclear Universidad de Sevilla, Apdo. 1065, E-41080 Sevilla, Spain
}

(Received 4 August 2005; published 12 July 2006)

\begin{abstract}
The implementation of a number-conserving formalism for pair-addition and pair-removal modes has impaired the possibility of developing an intuitive and self-consistent picture for collective pairing modes. In this paper, we show how a relaxation of this constraint leads to a unified model with true predictive power.
\end{abstract}

DOI: 10.1103/PhysRevC.74.014307

PACS number(s): 21.10.Re, 21.10.Dr, 21.60.Ev, 21.60.Jz

\section{INTRODUCTION}

The concept of collective pair-vibrational modes in nuclear systems was introduced in the middle 1960s and developed mostly in the decade that followed [1]. Ever since work began on this subject, it has often been stressed that a close analogy exists between these elementary modes of excitation and the more familiar surface vibrations of nuclear density [2]. However, in the pairing case, conservation of the number of particles keeps apart the modes associated with the addition of two nucleons to the reference state from those associated with the removal of a pair. As a consequence, the theoretical formalisms developed for the microscopic construction of the modes have led to dispersion relations where the pair addition and removal modes emerge as separate, independent solutions.

There is another fundamental difference between the treatment of pairing and surface modes that evolved from this situation. While it was easy to visualize what was "vibrating" in the case of a surface mode, no simple interpretation of any of the pairing vibrations was readily available. This aspect of the problem should not be overlooked. In fact, the intuitive character of the surface vibrations led to the development of a macroscopic model whose practical advantage over the microscopic description of the modes proved crucial, especially in the analysis of experimental data. Let us just mention, as an example, the simple macroscopic expressions for the coupling form factors that are used to describe the inelastic excitation of vibrational states [3]. While the development of a microscopic counterpart was a definite challenge and an important step in our understanding of nuclear structure (see, e.g., Ref. [4]), it is hard to envisage how the systematic accumulation of information on nuclear deformation parameters would have evolved if forced to rely exclusively on such a microscopic alternative.

Some years ago, concerted attempts were made [5,6] to introduce macroscopic form factors for the collective transfer of nucleon pairs (for a general review, see also Ref. [7]). These developments basically interpreted the pairing modes as oscillations across the mass partitions and assigned the role of a collective variable to the change in number of particles, $\delta A$. The prominent feature of this approach was a simplifying ansatz that related the pair transition density to the change of the saturated nuclear density with the number of nucleons $A$. This connection, in turn, provided a straightforward prescription for introducing the radial dependence of the transfer form factors. In this paper, we elaborate further on these ideas and show how it is possible to construct a self-consistent picture of pairing vibrations which incorporates a significant number of desirable features.

The contribution is organized as follows. In Sec. II, we review the experimental information that led to the concept of pairing vibrations in nuclei and introduce the particular point of view that we intend to exploit. The relevant microscopic and macroscopic aspects of the problem are given in Sec. III. As a consequence of these arguments, it becomes possible, in Sec. IV, to introduce a parameter-free, self-consistent pairing coupling constant. Section V gives a detailed description of the calculational scheme to be employed, and Sec. VI presents a brief discussion about the identification of those specific systems likely to have pair vibrations. The results of our analysis-including a comparison with experimental values-are shown in Sec. VII. Theoretical arguments for the $A$ dependence of the pairing energy term and its connection with the empirical parametrization introduced decades ago are presented in Sec. VIII. A brief summary and some conclusions close the paper in Sec. IX.

\section{FORMULATION OF THE PROBLEM}

Let us recall the practical procedure that reveals the existence of a vibrational-like structure involving nuclei which differ by $\pm 2, \pm 4, \ldots$ protons or neutrons with respect to a closed-shell system with a total number of particles $A$.

We first choose the position of the Fermi level $\lambda$ equidistant from the single-particle levels that correspond to the lastoccupied (with energy $\bar{e}_{h}$ ) and first-unoccupied states (with energy $\bar{e}_{p}$ ) at the shell closure, namely,

$$
\lambda=\frac{\bar{e}_{p}+\bar{e}_{h}}{2} .
$$

Then, using the nuclear binding energies $B$ and a suitably defined excitation energy scale,

$$
E\left(A^{\prime}\right)=B\left(A^{\prime}\right)-B(A)-\lambda\left(A^{\prime}-A\right),
$$

the ground states of the even systems of mass $A^{\prime}$ in the vicinity of the "reference" nucleus with mass number $A$ can be arranged as shown in Fig. 1, adapted from Ref. [8]. This particular illustration is for adding or subtracting pairs of neutrons around ${ }^{208} \mathrm{~Pb}$, but similar situations are encountered-also with proton 


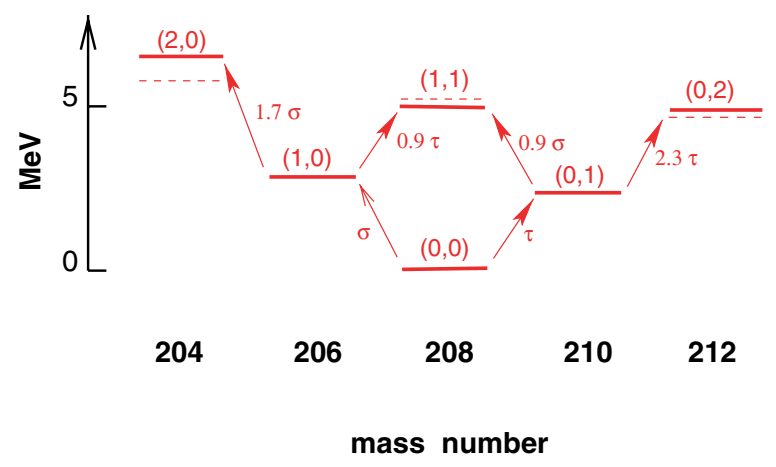

FIG. 1. (Color online) Level scheme for different isotopes of lead with an even number of neutrons in the vicinity of ${ }^{208} \mathrm{~Pb}$. The scale of energies has been defined as explained in the text. The quantities written next to the arrows give the cross sections for the observed $(t, p)$ and $(p, t)$ collective transitions measured in units of $\sigma$ and $\tau$. These quantities correspond to the basic processes of two-neutron pickup and stripping from the reference closed-shell nucleus. A pure harmonic picture would have the levels with number of removal and addition phonons $\left(n_{r}, n_{a}\right)$ satisfying $n_{a}+n_{r}=2$ to have the energies indicated by the dashed lines and the numbers $1.7,0.9$, and 2.3 to be $2.0,1.0$, and 2.0, respectively.

pairs - for many other systems in selected regions of the chart of nuclei (as we shall soon see).

The harmonic-like character of the resulting paircorrelation energy spectrum becomes evident in this representation, and the labels $\left(n_{r}, n_{a}\right)$ quoted next to each level correspond to the conventional interpretation of the states in terms of the number of pair-addition and pair-removal phonons involved. Note that, to complete the diagram, one extra level has been included which actually corresponds to an excited state of the reference nucleus.

In what follows, we strictly enforce the apparent left-right symmetry with respect to the closed shell displayed by the experimental data in Fig. 1 for the case of $\mathrm{Pb}$ (and similar diagrams for other systems). The resulting level scheme can be seen at the left-hand side of Fig. 2 . We then proceed to articulate a number-nonconserving description of the problem by "compressing" the schematic, symmetrized spectrum at the left of Fig. 2 into a set of levels identified only by the total

(a)

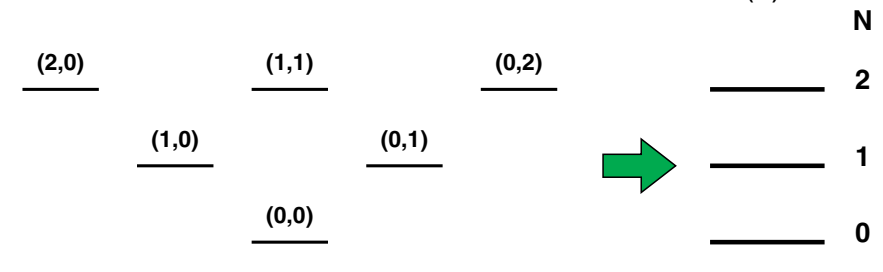

FIG. 2. (Color online) (a) Schematic representation of an energy diagram for pairing modes where symmetry between addition and removal modes as well as a strict harmonic character of the level scheme have been enforced. Levels are labeled by the number of quanta $\left(n_{r}, n_{a}\right)$ of the pair removal and addition modes. (b) Reduction of the previous scheme obtained by retaining only the information associated with $N=n_{a}+n_{r}$. number of phonons $N=n_{a}+n_{r}$, as shown to the right in Fig. 2.

The states of the resulting harmonic spectrum incorporate the combined action of the pair-addition and pair-removal creation operators, and therefore they no longer can be ascribed a definite number of particles. Actually, as one climbs up the new ladder of excited states, the particle content gets progressively richer. This is what one could qualitatively expect from an oscillation across the mass partitions whose amplitude $\delta A$ grows in mean-square value with the number of phonons $N$.

We now argue that while neither the pair-addition nor the pair-removal modes are amenable per se to a macroscopic description, a suitable combination of them may be. The way we are posing the problem is not obvious and/or beyond dispute, and therefore one cannot immediately judge the soundness of such a proposition. However, we will be able to show that, if one accepts this premise, the resulting formalism recovers the full formal analogy with the ordinary surface vibrations. Furthermore, adopting a macroscopic point of view makes it possible to define self-consistently the particlevibration coupling for the pairing modes. This yields an analytical expression for the coupling constant that governs the residual pairing interaction so that one can actually predict the correlation energy revealed by the experimentally measured nuclear masses.

We are well aware that in the course of this presentation we occasionally deviate from some conventional wisdom in the field (not that much, though). However, we note that the concept of "pairing"- not having a classical analog-is whatever we have made of it in terms of some model effective interactions and the experimental facts that they are meant to describe. From this point of view, one can hardly think of a better challenge than contributing to the understanding of the peculiar energy correlations revealed by the measured nuclear masses. The patterns of separation energies of single nucleons were, after all, the fundamental source of information that introduced the "pairing" concept in the discipline.

\section{MICROSCOPIC AND MACROSCOPIC ASPECTS}

Let us start by discussing the microscopic aspect of the problem. We recall that the standard Hamiltonian to treat pairing residual interactions is of the form

$$
\bar{H}_{p}=G P^{\dagger} P
$$

where $P^{\dagger}=\sum_{k>0} a_{k}^{\dagger} a_{\bar{k}}^{\dagger}$ is an operator that adds two particles in a combination of time-reversal states $k$ ( $\left.\equiv n_{k} \ell_{k} j_{k} m_{k}\right)$, $\bar{k}$ coupled to angular momentum zero. Given the numbernonconserving character of the proposed states, we here modify this expression and adopt

$$
H_{p}=\frac{1}{2} g Q^{\dagger} Q
$$


where $Q^{\dagger}$ is a generalized monopole one-body operator [7] of the form

$$
Q^{\dagger}=\frac{1}{\sqrt{2}} \sum_{k k^{\prime}>0}\left[q_{k k^{\prime}} a_{k}^{\dagger} a_{\bar{k}^{\prime}}^{\dagger}+q_{k k^{\prime}}^{*} a_{\bar{k}^{\prime}} a_{k}\right]
$$

In terms of the traditional pairing model, what we have basically established is a correspondence of the type $Q^{\dagger} \leftrightarrow$ $\left(P^{\dagger}+P\right) / \sqrt{2}$. The expression (3.3) allows for the presence of radial matrix elements that connect all the different single-particle orbitals that can couple to angular moment zero (naturally $n_{k}=n_{k^{\prime}}$, but also $\left.n_{k} \neq n_{k^{\prime}}\right)^{1}$. Of course, a radial dependence could be introduced in other operators, for instance, in $P^{\dagger}$.

The operator $Q^{\dagger}$ is now Hermitian and thus can be associated with a macroscopic variable whose diagonal matrix elements assume real values. It is precisely the macroscopic interpretation of the mode that provides, as we shall soon see, the radial dependence, units, and magnitude of the function $q(r)$ and define its matrix elements as

$$
q_{k k^{\prime}}^{*}=\int \Psi_{k}(\vec{r}) q(r) \Psi_{\vec{k}^{\prime}}(\vec{r}) d \vec{r} .
$$

The total Hamiltonian to describe our system along the lines we have just discussed can be written as

$$
H=H_{0}+H_{p}-\lambda N=\sum_{k} \epsilon_{k} a_{k}^{\dagger} a_{k}+\frac{1}{2} g Q^{2},
$$

where $H_{0}$ is the shell-model Hamiltonian, $N$ is the particlenumber operator, and $\epsilon_{k}=e_{k}-\lambda$ are the energies of the single-particle states referred to the Fermi level. The solution to this problem can now be constructed in complete analogy with the treatment for surface vibrations in the context of the random phase approximation (RPA). It is not necessary, in the context of this paper, to give in detail all the steps of the derivation. We limit ourselves to state that one can introduce a general phonon creating operator

$$
\Sigma_{v}^{\dagger}=\sum_{k, k^{\prime}>0}\left(A_{k, k^{\prime}}^{v} a_{k}^{\dagger} a_{\bar{k}^{\prime}}^{\dagger}+B_{k, k^{\prime}}^{v} a_{\bar{k}^{\prime}} a_{k}\right)
$$

and a correlated vacuum state $|\tilde{0}\rangle$ such that $\Sigma_{\nu}|\tilde{0}\rangle=0$, for all the states $v$ that diagonalize the Hamiltonian $H$. The quasiboson approximation then leads to an analytic expression for the coefficients in (3.6), namely,

$$
\begin{aligned}
A_{k k^{\prime}}^{v} & =\frac{\Lambda_{\nu} q_{k k^{\prime}}}{\hbar \Omega_{\nu}-\left(\epsilon_{k}+\epsilon_{\bar{k}^{\prime}}\right)}, \\
B_{k k^{\prime}}^{v} & =\frac{\Lambda_{\nu} q_{k k^{\prime}}^{*}}{\hbar \Omega_{v}+\left(\epsilon_{k}+\epsilon_{\bar{k}^{\prime}}\right)},
\end{aligned}
$$

where $\hbar \Omega_{v}$ is the energy of the mode and

$$
\Lambda_{v}=\frac{g}{2} \sum_{k, k^{\prime}>0}\left(\delta_{k m} \delta_{k^{\prime} m^{\prime}}-\delta_{k i} \delta_{k^{\prime} i^{\prime}}\right)\left(A_{k k^{\prime}}^{v} q_{k k^{\prime}}^{*}-B_{k k^{\prime}}^{v} q_{k k^{\prime}}\right) \text {. }
$$

\footnotetext{
${ }^{1}$ Even though the definition of $Q$ may bring to mind the BCS formalism, we stress that a quasiparticle transformation need not be invoked.
}

In this last formula, we introduce a notation in which the labels $m, m^{\prime}$ and $i, i^{\prime}$ are used to distinguish between states above and below the Fermi surface, respectively.

Exploiting these results, one arrives at the dispersion relation that yields the energy of the RPA roots, namely,

$$
\begin{aligned}
\frac{1}{g}= & \sum_{m, m^{\prime}>0} \frac{\left|q_{m m^{\prime}}\right|^{2}\left(\epsilon_{m}+\epsilon_{\bar{m}^{\prime}}\right)}{\left(\hbar \Omega_{v}\right)^{2}-\left(\epsilon_{m}+\epsilon_{\bar{m}^{\prime}}\right)^{2}} \\
& -\sum_{i, i^{\prime}>0} \frac{\left|q_{i i^{\prime}}\right|^{2}\left(\epsilon_{i}+\epsilon_{\bar{i}^{\prime}}\right)}{\left(\hbar \Omega_{v}\right)^{2}-\left(\epsilon_{i}+\epsilon_{\bar{i}^{\prime}}\right)^{2}} .
\end{aligned}
$$

Those familiar with the microscopic construction of vibrational modes may immediately recognize that the equation above now exhibits a mathematical structure entirely analogous to the dispersion relation used to solve for ordinary surface modes. This was not the case for the separate pairaddition and pair-removal states.

In practice, to obtain the energy $\hbar \Omega$ of the lowest correlated state, one needs an adequate basis of single-particle states (energies $\epsilon_{k}$, matrix elements $q_{k k^{\prime}}$ ) and, of course, the value of the coupling constant $g$. As is well known, the problem of solving the dispersion relation with the conventional formalism is often turned around so that one actually uses the experimentally known value of $\hbar \Omega$ to adjust or derive a value for $G$. Because of the "constant" matrix elements that enter in the definition of the operator $P$, the extracted values of $G$ depend strongly on the size of the single-particle space in which the calculation is made.

Let us now turn our attention to the macroscopic part of the problem. As we already anticipated, we take as a collective variable $\alpha$ the real amplitude $\delta A$ of an oscillation involving different mass partitions in the vicinity of the reference nucleus with mass number $A$. We stress, however, that the choice of scale is entirely arbitrary. Note that it does not matter if the pair vibration is associated with protons or neutrons since for a chain of isotopes or isotones, $\delta A$ is equal to either $\delta N$ or $\delta Z$. Following the prescription of Refs. [5,7], we write the change in the interaction energy resulting from a given value of the macroscopic variable $\alpha$ as

$$
\delta V=\frac{\partial V}{\partial A} \delta A=-\frac{\partial V}{\partial r} \frac{\partial R}{\partial A} \alpha
$$

where $R=r_{\circ} A^{1 / 3}$ is the radius of the reference system. Or, equivalently,

$$
\delta V=\kappa F(r) \alpha,
$$

i.e., proportional to the product of a monopole one-body field $F(r)$ and the macroscopic variable $\alpha$. An analogous procedure can be followed to express the variation in the density $\rho$ that accompanies a deviation from the reference mass $A$, as measured by $\alpha$,

$$
\delta \rho(r)=-\frac{R}{3 A} \frac{\partial \rho}{\partial r} \alpha
$$




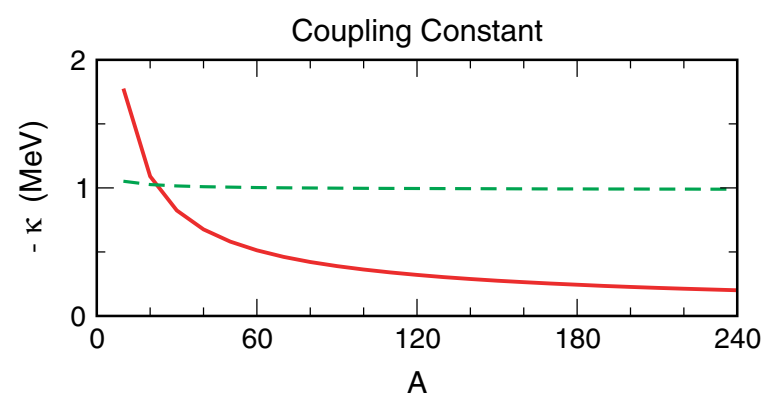

FIG. 3. (Color online) Dependence of the self-consistent coupling constant $-\kappa$ with the mass number $A$ (solid line). Dashed curve is proportional to $\kappa(A) A^{2 / 3}$.

\section{SELF-CONSISTENCY ${ }^{2}$}

Notice that the introduction of the constant $\kappa$ in Eq. (3.12) leaves us with a freedom of choice insofar as how much of the (physically invariant) product is carried by either $\kappa$ or $F$. This ambiguity is resolved and a point of contact between the macroscopic formulas and the microscopic approach discussed earlier is established by adopting a normalization of the field $F$ that allows us to relate $F$ with $Q$. To this end, we choose the field $F$ so that

$$
\langle F\rangle=\left\langle\sum_{k} F\left(r_{k}\right)\right\rangle=\int \delta \rho F d^{3} r=\alpha .
$$

Following this normalization, the residual interaction can be written as $H_{p}=\kappa F^{2} / 2$, and with this expression we identify $Q \Leftrightarrow F, g \Leftrightarrow \kappa$.

This correspondence, when applied to the normalization condition, leads to a self-consistent expression for the coupling constant,

$$
\kappa=4 \pi\left(\frac{R}{3 A}\right)^{2} \int_{0}^{\infty}\left(\frac{\partial \rho}{\partial r}\right)\left(\frac{\partial V}{\partial r}\right) r^{2} d r,
$$

which is a rather simple formula whose predictive power we proceed to test.

Using standard Woods-Saxon parametrizations for the density $\rho(r)$ and potential $V(r)$, the constant $\kappa$ (or $g$ ) can be calculated essentially "parameter free." It is clear from the above expression that keeping fixed the saturation values $\rho_{\circ}\left(\approx 0.16 \mathrm{fm}^{-3}\right), V_{\circ}(\approx 50 \mathrm{MeV})$, and $r_{\circ}(\approx 1.2 \mathrm{fm})$, the coupling constant depends only on the mass number $A$.

The function $-\kappa(A)$ is shown in Fig. 3 as a solid line, while the dashed curve results from multiplying $\kappa(A)$ by $-0.13 A^{2 / 3}$. Thus, with good accuracy

$$
\kappa(A) \approx-\frac{7.8}{A^{2 / 3}} \mathrm{MeV} .
$$

\footnotetext{
${ }^{2}$ Those who find the type of self-consistency arguments exploited in what follows unfamiliar may benefit from first reading Secs. 6.2 to 6.3 of Bohr and Mottelson [8], where they are used within the context of ordinary surface vibrations.
}

\section{CAlCUlation SCHEME}

The experimental quantities that are needed for our analysis can be directly extracted from the table of nuclear masses and the inferred values for the binding energies $B(A, Z)$. These include the already mentioned single-particle energies immediately above and below the Fermi surface,

$$
\bar{e}_{p}=B(A+1, Z+\sigma)-B(A, Z)
$$

and

$$
\bar{e}_{h}=B(A, Z)-B(A-1, Z-\sigma),
$$

from which the Fermi energy $\lambda=\left(\bar{e}_{p}+\bar{e}_{h}\right) / 2$ and the value of the gap $\Gamma=\left(\bar{e}_{p}-\bar{e}_{h}\right)$ are derived. Here $\sigma$ is an integer index that specifies if the calculation is made for neutrons $(\sigma=0)$ or protons $(\sigma=1)$. Also, the energies of the ordinary pairing modes,

$$
\begin{aligned}
\hbar \Omega_{ \pm}= & B(A \pm 2, Z \pm 2 \sigma)-2 B(A \pm 1, Z+\sigma) \\
& +B(A, Z) \mp \lambda,
\end{aligned}
$$

when combined, yield the experimental value of the phonon energy

$$
\hbar \Omega_{\exp }=\left(\hbar \Omega_{+}+\hbar \Omega_{-}\right) / 2 .
$$

To construct the modes microscopically, on the other hand, we need to collect a larger number of ingredients. Here one has to generate a table of single-particle states (discretized when they start being embedded in the continuum), construct the complete set of particle-particle and hole-hole matrix elements of the field, and specify a value for the coupling constant $\kappa$. Only with these elements in hand can one then proceed to solve the RPA-dispersion relation that yields the theoretical value $\hbar \Omega_{\text {th }}$.

Let us summarize with more detail. In order to compare the experimental quantities with the predictions of the selfconsistent model, we implemented a series of five computer programs that, in a completely automated sequence, perform the following tasks:

(i) Search the nuclear mass tables and extract the information necessary to construct $\bar{e}_{p}$ and $\bar{e}_{h}$, as defined above. These two quantities specify the Fermi energy $\lambda$ and the value of the gap $\Gamma$. Also, the quantities $\hbar \Omega_{+}$and $\hbar \Omega_{-}$are calculated which, in turn and as stated earlier, yield the experimental value of the phonon energy $\hbar \Omega_{\text {exp }}$.

(ii) Construct an ordered table of single-particle levels, containing for each orbital $k$ its quantum numbers $n_{k}, \ell_{k}$, and $j_{k}$ and the energy $\epsilon_{k}$ of the state referred to the Fermi surface. This is done following the prescription of the Lund group [9] and includes all the harmonic shells characterized by the main quantum number $0 \leq$ $N \leq N_{\text {max }}$. The two states above and below $\lambda$ are slightly adjusted to reproduce the experimental value of $\Gamma$ and, in all cases, the single-particle basis is truncated at the principal quantum number $N_{\max }=N_{\text {shell closure }}+3$.

(iii) Perform the numerical integration of Eq. (4.2) to return the constant $\kappa$ (or $g$ ) for the system under consideration. This is the same code used to draw Fig. 3. 


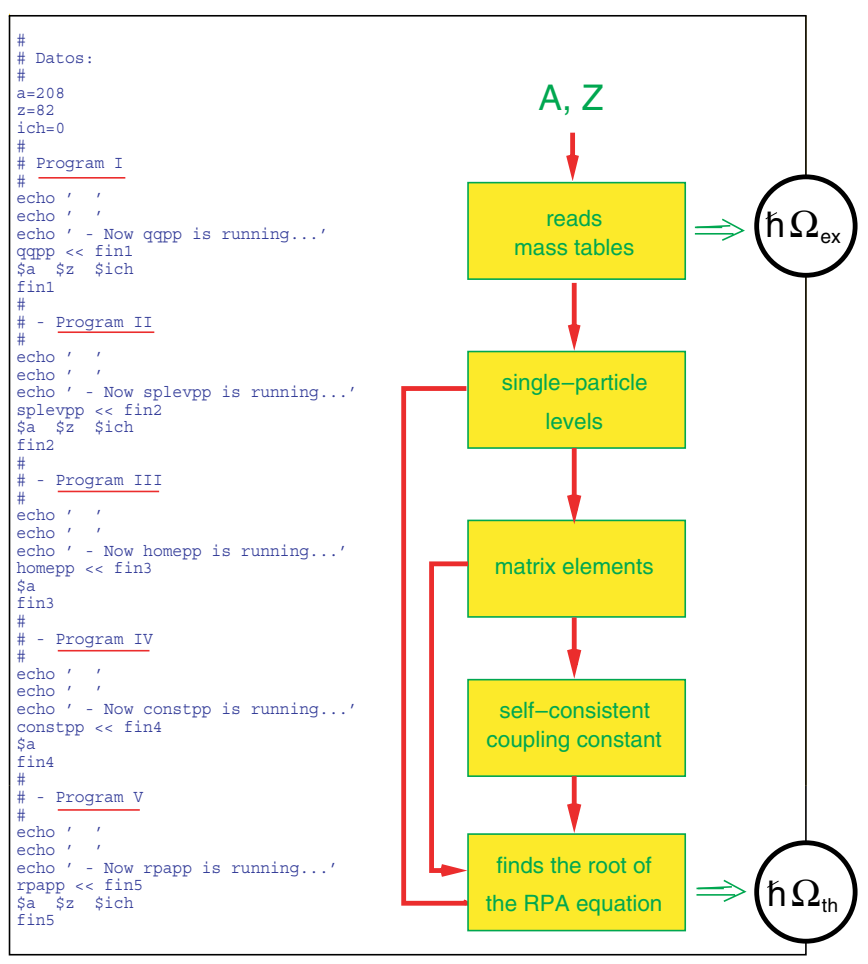

FIG. 4. (Color online) Overview of the calculation scheme used to generate our results. Given the values of $A$ and $Z$, five computer programs are executed in an automatic sequence. As a result, the values of $\hbar \Omega_{\exp }, \hbar \Omega_{\text {th }}$ are extracted, ready to be plotted and compared. For a brief explanation of the different blocks indicated in the figure (and their links) refer to the text. The flow diagram is superimposed onto the Unix script used to set into motion the entire sequence of calculations.

(iv) Construct the matrix elements of the field $Q$ between all the single-particle levels previously tabulated. Harmonic wave functions consistent with the Lund parametrization are used.

(v) Solve the RPA-like equations. The dispersion relation (3.10) is used to extract the lowest root (with energy inferior to $\Gamma$ ); this yields the value of $\hbar \Omega_{\mathrm{th}}$.

An overall view of the script that runs the five programs in a sequence is shown in Fig. 4, together with a block diagram of the operational procedure we just described.

Notice that to run the entire calculation, including the construction of the experimental quantities, one has to provide as external data only the numbers $A$ and $Z$. After the automated sequence of steps is completed, one can directly compare the theoretical and experimental values of $\hbar \Omega$.

Before we move on to present results for different nuclear systems, let us check the stability of the calculations as a function of $N_{\max }$. We take, as an example, neutron pair vibrations around ${ }^{208} \mathrm{~Pb}$ (i.e., the case displayed in Fig. 1). Table I shows the values obtained for $\hbar \Omega$ by changing the number $N_{\max }$ from 7 to 11 . This is $3 \pm 2$ major shells over the shell closure for the magic number 126 . Although an expected, monotonic change occurs, all the cases are within $20 \%$ of $\hbar \Omega_{\text {th }}=2.5 \mathrm{MeV}$, a significant degree of stability over a range of five major shells. We point out that the number
TABLE I. Convergence of results as a function of the size of the configuration space in which the RPA-like dispersion relation is solved. First column gives the maximum value of the harmonic-oscillator principal quantum number (the number of major shells included being $N_{\max }+1$ ). Middle column lists the number of components of the corresponding wave functions, and the last column gives the value obtained for the energy of the pairing mode.

\begin{tabular}{rcc}
\hline \hline$N_{\text {max }}$ & Wave function components & $\hbar \Omega_{\text {th }}(\mathrm{MeV})$ \\
\hline 7 & 62 & 2.9 \\
8 & 83 & 2.7 \\
9 & 109 & 2.5 \\
10 & 150 & 2.3 \\
11 & 198 & 2.0 \\
\hline \hline
\end{tabular}

of components of the collective wave function associated with such extension of the configuration space more than triples from 62 to 198. In what follows, we will only quote results calculated for $N_{\max }=N_{\text {shell closure }}+3$. A truncation at this level seems a reasonable compromise between having a sufficiently large configuration space and keeping away from a questionable handling of the continuum. In any case, to give a visual indication of the degree of convergence, the theoretical predictions will be, in Sec. VII, plotted with an "error" bar that gives the margin of change in the value of $\hbar \Omega_{\mathrm{th}}$ resulting from adding or subtracting two major shells.

The systems chosen are ${ }^{16} \mathrm{O},{ }^{40} \mathrm{Ca},{ }^{58} \mathrm{Fe}$, and ${ }^{208} \mathrm{~Pb}$ for protons and ${ }^{16} \mathrm{O},{ }^{40} \mathrm{Ca},{ }^{90} \mathrm{Zr}$, and ${ }^{208} \mathrm{~Pb}$ for neutrons. These nuclei are either double magic or, at least, magic in either neutrons or protons. To check the quality of the agreement over a narrow interval of mass numbers $A$, we have also added, to the systems listed above, a collection of isotones with $N=82$, namely, ${ }^{136} \mathrm{Xe},{ }^{138} \mathrm{Ba},{ }^{140} \mathrm{Ce}$, and ${ }^{142} \mathrm{Nd}$.

\section{IDENTIFYING PAIRING-VIBRATIONAL NUCLEI}

The list of nuclei given in the previous paragraph may appear at first sight as being short and somewhat arbitrary. We would like to stress, however, that with the inclusion of the last bunch of nuclei in the vicinity of ${ }^{136} \mathrm{Xe}$, we have covered all the mass regions where pairing vibrations manifest themselves.

This claim merits some explanation, and so we have introduced in this paper Figs. 5 and 6. The first of these figures displays the parameter

$$
\begin{aligned}
\Delta_{\exp }= & \frac{1}{4}[B(A-2, Z-2 \sigma)-3 B(A-1, Z-\sigma) \\
& +3 B(A, Z)-B(A+1, Z+\sigma)],
\end{aligned}
$$

calculated for a large number of heavy nuclei and plotted as a function of the mass number $A$. The values are computed automatically reading the electronic mass tables, and the result is familiar to most nuclear physicists since the distribution $\Delta_{\text {exp }}(A)$ is included in many standard textbooks.

After consulting with many colleagues, however, we concluded that the information contained in the upper frame of Fig. 6 is not as well known. Here, we display the values of 


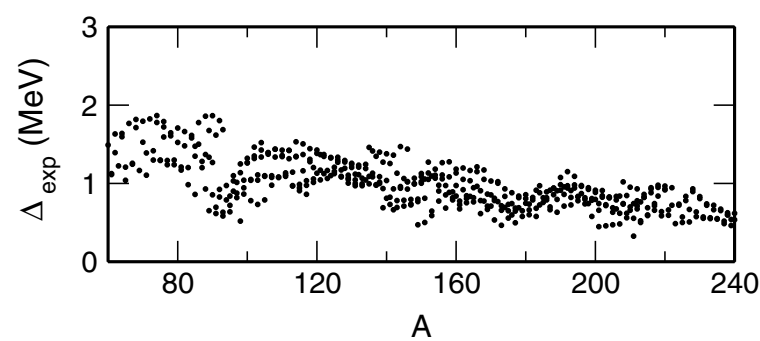

FIG. 5. Dependence of pairing parameter $\Delta_{\exp }$ as a function of mass number $A$.

$\hbar \Omega_{\exp }(A)$ calculated according to Eq. (5.4) and for the same collection of nuclei [the numbers were generated, in fact, together with those of $\left.\Delta_{\exp }(A)\right]$.

Notice that the experimental values of $\Delta$ plotted in Fig. 5 could not possibly be used to identify the rather select set of nuclei where pairing vibrations manifest themselves. In the vast majority of nuclear systems, in fact, the absence of a substantial gap separating the occupied from the unoccupied single-particle levels makes the BCS conditions prevail and superfluidity sets in.

The experimental values of $\hbar \Omega$, on the other hand, put into evidence the inherent advantage of a representation that neatly separates one situation from the other. Recalling that the values of the static and the dynamic surface deformation parameters $\beta$ are usually comparable whereas it is the excitation energy
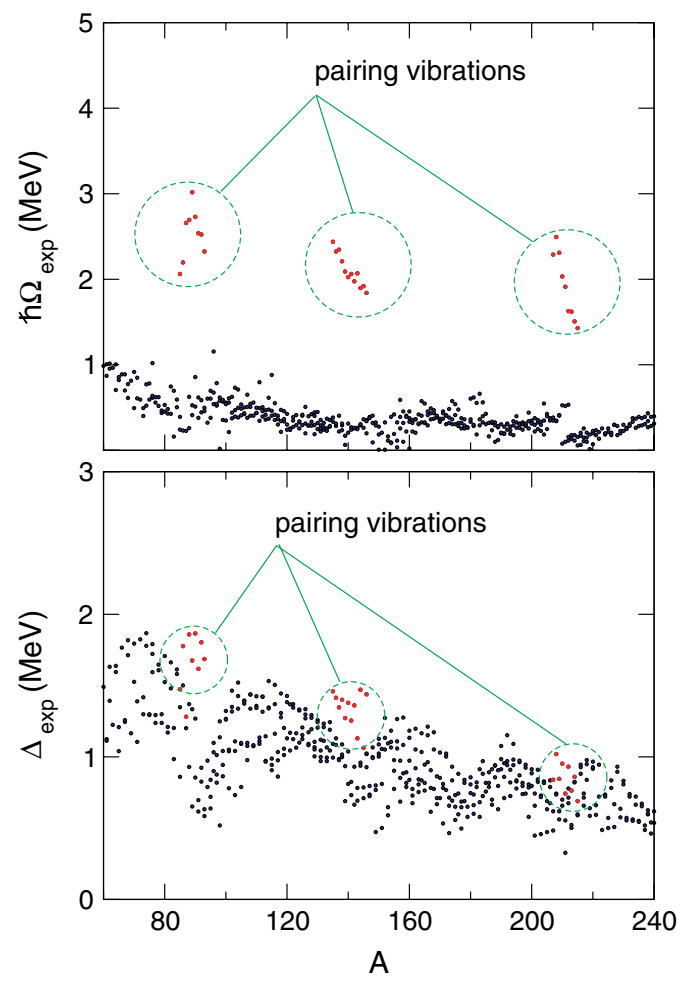

FIG. 6. (Color online) Top: dependence of the experimental values of $\hbar \Omega$ as a function of $A$. Bottom: same as above, but for the pairing parameter $\Delta$. Points inside the dashed circles correspond to pairing vibrations. scale that rather sets apart ordinary vibrational and rotational modes, this should not come as a complete surprise.

For the sake of clarity, we have chosen here to display the distributions of $\hbar \Omega_{\exp }$ and $\Delta_{\exp }$ for systems with $A \geqslant 60$. We stress, however, that there is no problem whatsoever with using our conceptual framework for lighter nuclei. (In fact, we will include in our comparison with experimental data the proton and neutron pairing vibrations for the nuclei of oxygen and calcium and display the results in the full range of $A$.)

We just said above that the values of $\Delta_{\exp }(A)$ for pairing vibrations blend, unfortunately, too well with the rest of the distribution to serve as an identifying marker. With the benefit of hindsight provided by the top of Fig. 6, one can see that the experimental values of $\Delta$ for this class of nuclei actually lie systematically somewhat higher than those for superfluid systems. (This is the purpose of the "bubbles" introduced in Fig. 6.) The magnitude of $\Delta_{\exp }$ and its overall $A$ dependence is nevertheless practically the same. We recommend reading this paper online since color in the figures makes it easier to realize this and other points.

\section{RESULTS}

The results of the calculations are collected in Fig. 7 where the theoretical (circles) and experimental (crosses) values of $\hbar \Omega$ are plotted as a function of $A$. For the values $A=16,40$, and 208 we have shifted slightly to the left (right) the points that correspond to the proton (neutron) cases. Notice that there is essentially only one theoretical prediction for $A=16$ and $A=40$. This is understood because the theoretical coupling constant (and the basis of single particles and matrix elements) is the same for protons and neutrons in ${ }^{16} \mathrm{O}$ and ${ }^{40} \mathrm{Ca}$. There are of course two experimental values which, in any case, do not differ by much. One has, on the other hand, two theoretical predictions (and two experimental numbers to compare with) in the case of ${ }^{208} \mathrm{~Pb}$, because even if the abscissa and coupling constant are the same, the microscopic calculations for protons around shell closure $Z=82$ and neutrons around $N=126$ are entirely different.

To give a visual indication of the degree of convergence with the size of the configuration space, the theoretical predictions in Fig. 7 are plotted (as mentioned earlier) with "error" bars; these indicate the range in the values of $\hbar \Omega_{\text {th }}$ resulting from adding to or subtracting from the single-particle basis two major shells. We note that the experimental numbers, extracted from high-precision measurements listed in the the mass tables, are virtually error free in the plotting scale.

We judge the overall agreement between theory and experiment to be very good. Not only is the expected trend to obtain larger excitation energies for the lighter nuclei reproduced, but also local deviations from a smooth behavior are quite accurately described. This is a more stringent test than merely obtaining the average $A$ dependence of the pairing parameter $\Delta$, which will be done below by exploiting dimensional arguments that link it to that of Eq. (4.2). One easily estimates the theoretical values of $\Delta, \Delta_{\text {th }}$, from these calculations, since the correlation energy is related to $\hbar \Omega$ and the value of the gap $\Gamma$ at the Fermi surface by $\Delta=\Gamma-\hbar \Omega$, both theoretical 


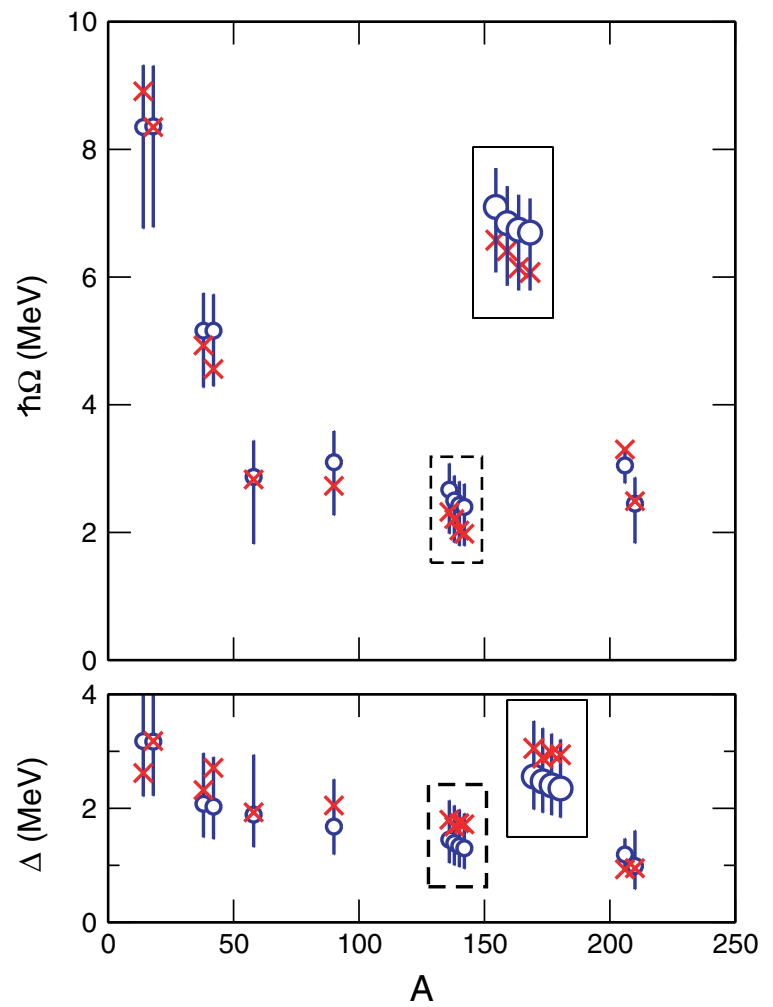

FIG. 7. (Color online) Energy of the pairing modes $\hbar \Omega$ as a function of $A$ for the different systems quoted in the text. Whenever the value of $A$ corresponds to systems where both proton and neutron pair vibrations have been calculated $(A=20,40,208)$, the theoretical (circles with error bars) and experimental (crosses) values of $\hbar \Omega$ for the proton systems have been slightly displaced to the left. Neutron systems have been displaced to the right by the same amount. The meaning of the error bars on the theoretical values is explained in the text. Insets show in more detail the results displayed inside the dashed-line boxes.

and experimental. (The values of this quantity in the range of $1-3 \mathrm{MeV}$ also indicate that the agreement shown in Fig. 7 is by no means trivial). The correct trend obtained for the energies of the $N=82$ isotones (shown in detail in the insets) could have been better reproduced by changing $N_{\max }$ by one unit. This we have not done, since at this stage and in our opinion, it does not make sense to optimize any particular agreement. Also because, as stated earlier, we wanted all the points displayed in the figure to emerge from a common calculation scheme (cf., Fig. 4) without any adjustments whatsoever. Keep in mind that the proposed procedure only requires as input data the knowledge of the mass and charge numbers of the system considered.

\section{THE FIFTH TERM OF VON WEIZSÄCKER'S SEMIEMPIRICAL BINDING ENERGY FORMULA}

It was mentioned earlier that setting a scale for our collective variable is entirely arbitrary. One could take, for instance, $\alpha \leftrightarrow \delta A / A$. This would lead to another expression of the coupling constant $\kappa$ and, in particular, to a different $A$ dependence. Such choice would, of course, not matter when comparing the results with experimental information, as long as one maintained overall consistency.

The identification of the macroscopic variable $\alpha \leftrightarrow \delta A$, however, is especially convenient because it establishes a direct link between the $A$ dependence of the pairing parameter and that of the coupling constant $\kappa$. In fact, one can obtain the mass dependence of $\Delta$ using lines of reasoning analogous to those successfully employed to infer, for instance, the $A$ dependence of the excitation energies of the giant-dipole or the giant-quadrupole resonances [8]. To this end, we first argue that the high degree of convergence put into evidence by the small error bars displayed in Fig. 7 makes it possible to retain, for the sake of argument, only the major shells directly above and below the Fermi surface. Then we note that details of the subshell energy splittings (and the actual ordering of the orbitals) cannot influence the general characteristics of the results. Thus, in a strict degenerate limit, we have

$$
\begin{aligned}
\frac{1}{\kappa} & =\left(\sum_{k k^{\prime}} q_{k k^{\prime}}^{2}\right) \frac{\Gamma}{(\hbar \Omega)^{2}-\Gamma^{2}} \\
& \approx \bar{q}^{2} 2 d \frac{\Gamma}{\Delta^{2}-2 \Gamma \Delta} \approx-\bar{q}^{2} d \frac{1}{\Delta},
\end{aligned}
$$

or, otherwise,

$$
\Delta \approx-\bar{q}^{2} d \kappa .
$$

Into this expression enters the square of a characteristic value $\bar{q}$ of the matrix elements of the field multiplied by the shell degeneracy $d$, both computed around the Fermi level. The mass dependence of the former follows from

$$
\bar{q}^{2} \approx\left[\frac{V_{0}}{4} \frac{1}{A} \frac{1}{\left(8 A^{-2 / 3} \mathrm{MeV}\right)}\right]^{2} \propto A^{-2 / 3}
$$

and just compensates with that of $d \approx A^{2 / 3}$ (cf., e.g., Ref. [8]). It follows from these considerations that $\Delta(A)$ has the same dependence with the total number of nucleons as $-\kappa(A)$. An inspection of Fig. 3 supports this assertion and yields a multiplicative factor of about 5 , which is, indeed, on the order of $\left[V_{0} /(30 \mathrm{MeV})\right]^{2}$.

An essentially equivalent statement is that a theoretical argument establishes that the $A$ dependence of the pairing contribution to the semiempirical mass formula should go as $A^{-2 / 3}$.

There is no universal agreement on a functional form for the pairing term in von Weizsäcker's binding-energy formula. The analytic expression is, after all, regarded as a semiempirical formula. Let us just simply recall that the textbook of Eisenberg and Greiner [10] gives for even- $A$ systems $\left|B_{p}\right|=a_{p} A^{-1 / 2}$, while Krane's volume [11] quotes $\left|B_{p}\right|=a_{p} A^{-3 / 4}$. We note that our power law lies just in between those parametrizations. The bottom part of Fig. 6 confirms that not only the $A$ dependence but also the absolute magnitude of $\Delta$ is correctly predicted.

\section{CLOSING REMARKS}

To summarize, the pairing picture we have introduced presents the following characteristics: 
(i) It is amenable to a macroscopic interpretation.

(ii) It restores the formal analogy between the mathematical expressions used to microscopically construct pairing and surface vibrations.

(iii) A self-consistent expression for the pairing coupling constant (the particle-vibration vertex) can be found.

(iv) The surface-peaked radial dependence of the field leads to a reasonable stability in the results of the calculations with respect to the size of the configuration space.

(v) It is able to predict the magnitude of pairing correlations revealed by the experimental measure of nuclear masses with remarkable precision.

(vi) The $A$ dependence of the pairing interaction energy that follows from the analytic expression of the coupling constant agrees perfectly with decades-old empirical parametrizations.

One could also add to this list the fact that the formalism supports (and, conversely, gets support from) macroscopic calculations of pair-transfer processes that, done more than a decade ago, showed promise as an alternative to the standard microscopic approach.

Calculations with a more realistic single-particle basis of states and matrix elements and incorporating a better handling of the continuum should improve the quality of this analysis. As we know, this is what happened in the study of surface vibrations. However, we also know that the basic understanding of these modes (as explained, for instance, in Ref. [8]) did not change radically as a consequence of the gradual incorporation of more sophisticated techniques. We have checked that the general conclusions of this analysis do not depend on our particular choices of configuration space and nuclear parameters. Thus, hopefully, a similar situation may evolve from the investigation of pairing vibrations along the lines suggested here.

We would like to conclude with a quote by Aage Bohr from his Nordita Lecture Notes of 1979 [12] that we find relevant in the context of this paper:

"As we have seen, the combination $a^{\dagger} a$ is associated with the one-particle operators such as the density $\rho(\vec{r})$ or the potential $V$. When viewed in this way there seems to be a natural place for densities and potentials associated with the combinations $a^{\dagger} a^{\dagger}$ and $a a$. However, you may then argue that since these densities do not conserve the particle number $(N)$ they have no natural role in the description of a system with a fixed number of particles, like a nucleus (e.g. the expectation value for any state or ensemble with fixed number of particles is zero, $\left.\left\langle N\left|a^{\dagger} a^{\dagger}\right| N\right\rangle=0\right)$.

Retrospectively one might be tempted to say that this attitude held up the development of the present subject for some thirty years."

\section{ACKNOWLEDGMENTS}

Support is acknowledged from FEDER funds and the Ministry of Education and Science under project numbers FIS2005-01105, FPA2005-04460.
[1] A. Bohr, in Comptes Rendus Congrès International de Physique Nucléaire, edited by P. Gugenberger (CNRS, Paris, 1964), Vol. I, p. 487; A. Bohr, in Nuclear Structure, Dubna Symposium (IAEA, Vienna, 1968), p. 179; D. R. Bès and R. Broglia, Nucl. Phys. 80, 289 (1966).

[2] R. Broglia, O. Hansen, and C. Riedel, in Advances in Nuclear Physics, edited by M. Baranger and E. Vogt (Plenum, New York, 1973), Vol. 6, p. 287.

[3] R. Broglia and A. Winther, Heavy Ion Reactions (AddisonWesley, Redwood City, CA, 1991).

[4] R. Broglia, C. H. Dasso, G. Pollarolo, and A. Winther, Phys. Rep. 48C, 351 (1978).

[5] C. H. Dasso and G. Pollarolo, Phys. Lett. B155, 223 (1985).

[6] C. H. Dasso and A. Vitturi, Phys. Rev. Lett. 59, 634 (1987).
[7] C. H. Dasso, J. Fernandez-Niello, M. Gallardo, S. Land-owne, R. J. Liotta, P. Lotti, M. Lozano, G. Montagnoli, M. Nagarajan, H. M. Sofia, A. Stefanini, T. Vertse, A. Vitturi, H. H. Wolter, and L. Zybert, Ital. Phys. Soc. Conf. Proc. 18, 5 (1989).

[8] A. Bohr and B. Mottelson, in Nuclear Structure (Benjamin, Reading, MA, 1975), Vol. II, p. 646.

[9] S. G. Nilsson, C. F. Tsang, A. Sobiczewski, Z. Szymánski, S. Wycech, C. Gustafson, I.-L. Lamm, P. Moller, and B. Nilsson, Nucl. Phys. A131, 1 (1969).

[10] J. M. Eisenberg and W. Greiner, Nuclear Models (North Holland/American Elsevier, Amsterdam, 1975).

[11] K. S. Krane, Introductory Nuclear Physics (Wiley, New York, 1988).

[12] A. Bohr, J. M. Leinaas, and P. Minnhagen, Nordita Lecture notes, 1979, preprint 79/13 (unpublished). 